\title{
HOSPITAL-ACQUIRED INFECTIONS
}

\author{
THE NATIONAL AGENDA \\ Sue Partridge, MRCPath
}

It is difficult to write on the subject of hospital-acquired infections (HAI) without sounding clichéd. The subject is the focus of considerable media attention, and reference was made to it at the recent Party Conferences. It would be easy to think, based on these sources, that the problems were due to:

- dirty hospitals

- dirty doctors' white coats (or should I say doctors' dirty white coats?)

- healthcare workers not washing their hands

Clearly the situation is more complicated than that. Hospitals should be clean for all sorts of reasons, and the role of fomites in transmission of infection can be significant - an extreme example would be the dirty commode. The proposal for an annual 'deep clean' of every ward may be laudable, but it is what happens every day that is more important.

Items of clothing have been shown to 'harbour' bacteria, but not demonstrated to be responsible for the transmission of infection. It makes sense, however, for staff to wear clothing which can be regularly and effectively cleaned, and the style of which facilitates good hand decontamination. After all, do you really want to go around with a patient's body fluids on your clothes?

Hand decontamination is a bit of an infection control cliché (in that it frequently forms part of discussions about infection control). It is, however, of fundamental importance.

The fact is, however, that we face a fundamental problem in the control of infection, because hospitals are intrinsically high risk in this regard. We bring together a group of vulnerable people, with underlying conditions, many of whom are elderly, and do things to them which increase their vulnerability to infection. How many patients escape without a cannula? What about urinary catheters? Surgery? The situation is compounded by the administration of antibiotics. These select out resistant organisms, make patients more vulnerable to the acquisition of 'hospital' bugs, and in the case of Clostridium difficile significantly alter the bowel microflora creating an environment ideal for proliferation of either pre-existing or acquired $C$. difficile organisms.

It would be easy to be defeatist about this, but we must not. The national and local figures show a significant increase in cases of $C$. difficile infection, even allowing for improved awareness and increased testing. You know what a miserable condition this is for patients, as well as being associated with death in some cases. Do we just accept it as 'inevitable', or that we are merely 'part of the national trend'? I would argue definitely not. We have responsibility for our patients, to give them the best available and appropriate care. Part of this must be to do what we can to reduce the chance of them acquiring an HAI. This means judicious use of antibiotics, getting rid of devices such as cannulae as soon as possible, and ensuring that in all contacts with patients control of infection procedures are rigorously followed.

We must be prepared to challenge others if their practice is not 'best practice'. This may in the future involve audits of antibiotic use and of hand decontamination (for example). We must also be prepared to be challenged ourselves, if our own practice is suboptimal.

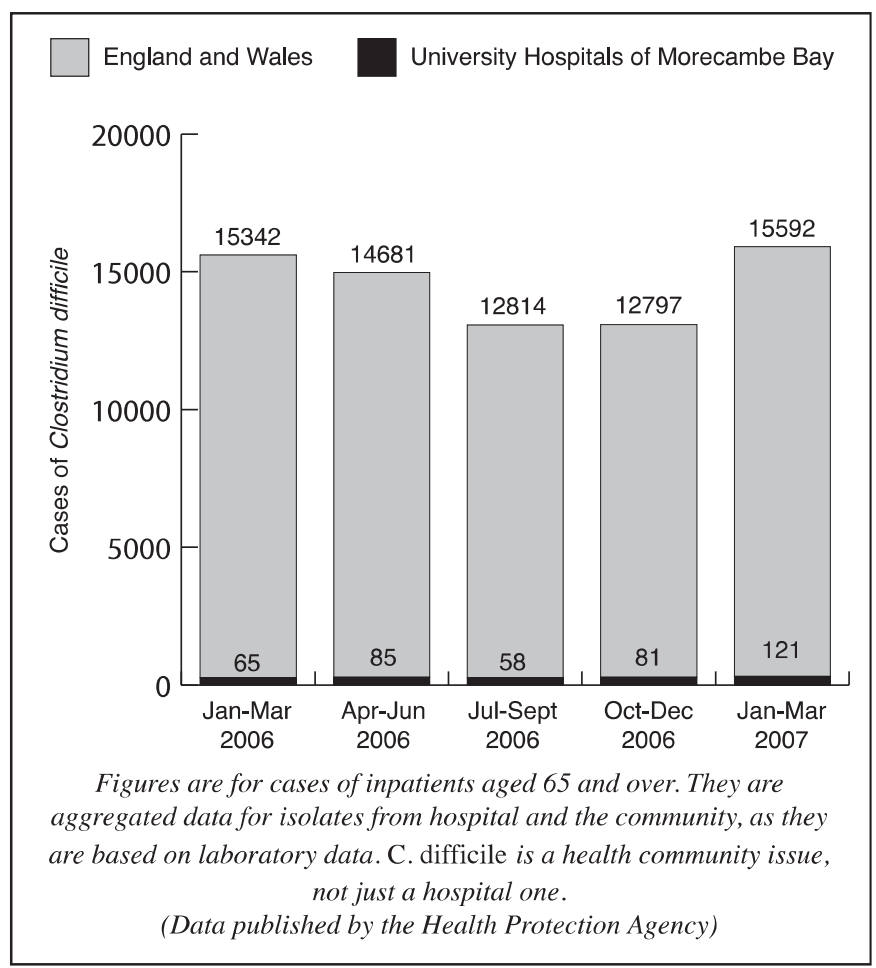

National agendas require me to submit increasing amounts of data on the rates of HAI. National and local targets for numbers of cases of methicillin resistant Staphylococcus aureus (MRSA) bacteraemia and $C$. difficile infection already exist. They are not going to go away. Tools such as Root Cause Analysis (RCA) are now mandatory in the investigation of MRSA bacteraemias (and are shortly to be introduced for $C$. difficile). We can either regard this as a pointless task to be dealt with as quickly and superficially as possible, or we can use it to generate information about our local cases, and use that information to focus our efforts to prevent HAI. These RCAs are not the province of the infection control team. They have to be completed by the staff 'on the ground'. At the moment they are undertaken by 
the modern matrons working with the ward-based nurses. The exercise will be made more valuable by the input of the medical staff. I shall be encouraging the modern matrons to involve medical staff, so be prepared for this. The numbers of MRSA bacteraemias in our trust are relatively low, so each consultant will only be involved in a few cases each year.
We must take the issue of HAI seriously. Some trusts report MRSA bacteraemias as 'Serious Untoward Clinical Incidents'. They should certainly be regarded as such.

\section{THE LOCAL PLAN Elizabeth Heffernan, RGN}

Over the past 12 months, Ward 6 at Furness General Hospital has taken a special interest in patients with Clostridium difficile due to an outbreak on the ward back in 2006. We have reviewed our clinical practices and focused on becoming more assertive regarding the review of antibiotic therapies and encourage the medical teams to consult between themselves and the microbiologist team for their input.

We feel it is very important to maintain good communication lines with patients and carers to inform them of such infections - ensuring information leaflets are passed on and allowing time for questions to be asked and answered - as it can be a very difficult time for families, especially as it can be fatal. The whole ward is committed to striving for good practice. As a ward manager, I demand good practice in hand washing from all my team and in turn I expect them to coach patients and relatives how important hand washing is in patients with $C$. difficile. This is something we all need to constantly police and encourage. The ward has also introduced the distribution of hand wipes before the serving of all meals; again this was implemented by Ward 6 and has been taken up by the whole division. The introduction of the use of the Bristol Stool Chart, which is an indicator of type of bowel movement that can show the patient's condition improving, is now being used by the Medical Division and is considered to be good practice.

As a ward manger, part of my role includes cleanliness and I see this as a high priority with all my team being fully committed. Also included are the domestics; we have a ward work plan which includes the thorough cleaning of our sixbedded bays. I am always exploring areas for improvement and at present I am investigating the possibility of changing my domestics' shift pattern to enable cleaning to take place twice a day in infected areas.

Ward 6 has also introduced disposable washbowls, which have been a great success, so much so they have been introduced across the division. The aim of the bowls is to reduce cross infection.

Ward 6 is always striving to implement new ideas and show good practice, as this has an impact on the patients' stay, giving them a good experience.

As everybody is aware, patients with $C$. difficile have frequent bowel movements. This can lead to an increase in soiled clothing. After a lot of investigation, we have introduced a relative's bag, in which they can take soiled linen home and place it directly into their washer. As the stitching in the bag dissolves, the families do not have to touch the linen - this is an area that has caused concern from relatives.

All staff on Ward 6 have learnt, and continue to learn, regarding $C$. difficile; but it is with their commitment and focus that $I$ have been able to make changes and strive for best practice. It is and will continue to be a challenge, but with good team work great results can be achieved and with this comes satisfaction that patients make a full recovery from a debilitating illness. 